\title{
Apropiación y control social en un centro comercial de Santiago: prácticas socioespaciales y significaciones adolescentes
}

Miguel Pérez. Universidad de California, Berkeley, CA. Estados Unidos. Rodrigo Salcedo. Pontificia Universidad Católica de Chile, Santiago, Chile. Gonzalo Cáceres. Pontificia Universidad Católica de Chile, Santiago, Chile.

RESUMEN | La crítica intelectual, tanto en Norteamérica como Latinoamérica, ha tendido a mostrar el mall como un espacio privado sobrecontrolado, socialmente homogéneo e indiferente al contexto urbano que lo rodea. En Chile tales narrativas implicaron, además, un cuestionamiento al modelo neoliberal implantado por la dictadura militar, hecho que no logró amilanar el éxito que tuvo el mall entre los ciudadanos. En este artículo, mediante un análisis cualitativo y etnográfico de las prácticas y significaciones de los usuarios adolescentes sobre el Mall Plaza Vespucio, debatiremos algunos de los discursos más recurrentes de la crítica académica, afirmando que para los jóvenes: (i) el mall, más que cerrado e indiferente a su contexto urbano, es parte de un sistema que incluye diversos espacios; (ii) las distinciones adentro/afuera y público/privado no son relevantes; (iii) los niveles de control social al interior del mall son tolerados según emerjan prácticas de resistencia y adaptación.

PALABRAS CLAVE | espacio público, consumo, cultura urbana, sociología urbana.

ABSTRACT | Intellectual critique, both from North and Latin America, has described malls as tightly controlled, socially homogeneous private spaces that are indifferent to their urban context. In Chile, this also implied a critique of the neoliberal model established during the dictatorship, which however, did not deny the mall's success among citizens. In this article, through a qualitative and ethnographical analysis of practices and significations of teenagers in the Plaza Vespucio Mall, we will discuss some of the most common discourses of the intellectual critique by asserting that for adolescents: (i) the mall, rather than being enclosed and indifferent to the larger urban context, is part of a system including different types of spaces; (ii) the private / public; inside / outside distinctions are not relevant; and (iii) the level of social control in the mall is tolerated insofar as practices of adaptation and emerging resistance.

KEY WORDS | public space, consumption, urban culture, urban sociology.

Recibido el 19 de enero de 2011, aprobado el 31 de mayo de 2011.

E-mail: Miguel Pérez, mperezahumada@berkeley.edu|E-mail: Gonzalo Cáceres, gacacere@uc.cl| Rodrigo Salcedo, rsalcedo@uc.cl

El artículo es tributario de la tesis para optar al grado de Magíster en Desarrollo Urbano (Pontificia Universidad Católica de Chile) desarrollada por el autor principal de este texto, la cual se tituló "Cuando los indeseados se congregan en el mall. Prácticas socioespaciales de adolescentes en un espacio semipúblico". A su vez, el estudio se enmarcó en el proyecto Fondecyt № 1085276, "No hay mall que por bien no venga. Efectos urbanos y re-significaciones culturales en una subcentralidad de Santiago (el caso de Mall Plaza Vespucio 1990-2007)". 


\section{Introducción}

El shopping mall aparece en EE. UU. a mediados del siglo veinte y es una expresión del proceso de suburbanización de la sociedad norteamericana. Mientras los centros urbanos de la posguerra se despoblaban aceleradamente y la ideología antiurbana expandía su feligresía, los desarrolladores inmobiliarios les prometieron a los compradores suburbanos la preservación de la rentabilidad del suelo y una utópica comunidad de iguales (Fishman, 1987). Para sus primeros diseñadores, los centros comerciales fueron imaginados desde la posibilidad de conjugar consumo, vida cívica y la emergencia de una colectividad en un espacio cerrado (Amendola, 2000).

Apenas se inició la dispersión de centros comerciales hacia destinos urbanos y suburbanos, las opiniones no se dejaron esperar. Desde una orilla obsecuente, planificadores urbanos (Welch, 1948), desarrolladores inmobiliarios (Nichols, 1948), arquitectos (Gruen, 1943) y especialistas en marketing (Merts, 1949) ensalzaron las posibilidades que brindaban los nuevos establecimientos para un país cada vez más automovilizado. No pasó demasiado tiempo antes de que los atributos y deméritos de lo que muy pronto sería uno de los principales íconos del sueño americano concitaran el interés del conjunto de las ciencias sociales, lo mismo que de las humanidades críticas. La americanización del mundo explica por qué también el cine y la literatura han incorporado el centro comercial a sus respectivas narrativas, destacándose, por ejemplo, la mordacidad de George Romero o Woody Allen y las alegorías de José Saramago.

Debido a la influencia ejercida por los centros comerciales, críticos y defensores han generado interpretaciones extremadamente variadas. Por ejemplo, contamos con estudios que los han analizado y descrito como lugares expresivos de paradojas y contradicciones modernas que se producen en masa para, al igual que otros objetos, ser consumidos (Sack, 1988); espacios que evocan antiguas utopías comunitarias y donde se producen fantasías colectivas (Goss, 1999); ciudades artificiales, deslocalizadas, protegidas y acondicionadas para el consumo (Amendola, 2000) o simplemente como "no-lugares" (Augé, 2004).

Sin perjuicio de aportaciones específicas, entre las narrativas que más predominan a la hora de caracterizar el mall, algunas lo destacan como un espacio socialmente homogéneo y segregador (e.g. Connell, 1999; Staeheli \& Mitchell, 2006); otras como un "espacio isla", encerrado e indiferente al contexto urbano (e.g. Ritzer 2003; Sarlo, 1994); o como un espacio súper vigilado y donde el control social se lleva al extremo (e.g. Lofland, 1998; Mugan \& Erkip, 2009). Además, en América Latina, pese a que algunos centros comerciales de México y Brasil fueron diseñados en la década de los 60, la academia ha tendido a presentar a los shopping malls ya sea como ejemplos y metáforas del neoliberalismo y la globalización (De Mattos, 1999; Ciccolella, 1999) o como una penetración de la cultura estadounidense (Gallardo, 1994), totalmente contradictoria con el ánimo europeizante y citadino de lo que sería la intelectualidad subcontinental. 


\section{El mall como espacio socialmente homogéneo y segregador}

Dado el interés del centro comercial por desincentivar la concurrencia de pobres, minorías y, en algunos casos, adolescentes; y, en el contexto norteamericano, por su localización alejada del transporte público, el mall se constituye, para muchos académicos, en un aparato socioespacial excluyente y segregador (Rile Hayward, 2001; Connell, 1999; Judd, 1995; Matthews, Taylor, Percy-Smith \& Limb, 2000). Para los que suscriben esta posición, el mall sería un espacio que, por razones comerciales, valoraría desmedidamente la homogeneidad social, evitando a los consumidores burgueses el estrés del encuentro con el 'otro' social. Lo que se buscaría, entonces, es generar una comunidad (de iguales), lo que reduciría el potencial público y político del espacio (Staeheli \& Mitchel, 2006).

\section{El mall como isla}

Una parte relevante del carácter segregador del mall tiene que ver con su capacidad para distanciarse tanto material como simbólicamente de su entorno físico. Esta característica ha sido destacada por diversos autores. Así, para Ritzer (2003), los malls pueden ser descritos como "islas" en las que se agudizan las tendencias de hiper racionalización y burocratización de la sociedad.

Para el ámbito latinoamericano, Sarlo (1994) sostuvo originalmente que el shopping center, con los atributos de un espacio neutro, desterritorializado y deshistorizado, se instaló en las ciudades sin consideración del contexto cultural y urbano que lo rodeaba, ignorando cualquier tipo de preexistencia. En un diagnóstico lapidario, Sarlo llega a decir que, "frente a la ciudad real, construida en el tiempo, el shopping ofrece su modelo de ciudad de servicios miniaturizada; la que se independiza soberanamente de las tradiciones y de su entorno" (pp. 18-19). Este cuestionamiento al mall se ve transformado y moderado a partir de las preguntas que Sarlo (2009) se formula, quince años más tarde, respecto de las razones de su éxito, llevándola a un reconocimiento, al menos parcial e ideológico, de las funciones integrativas y de socialización que el centro comercial ofrece (p. 7).

Ubicua antes que particular, la crítica de "excesivo enclaustramiento" aparece, en la arquitectura y el urbanismo norteamericanos, explicando las razones de la quiebra de diversos malls tipo "caja de zapatos" hacia fines de los años 1990 (Lieberman, 2004; Thomas, 2006; Perry, 2010).

\section{El mall como espacio hiper vigilado}

Diversos académicos han destacado las medidas de seguridad y los sistemas de vigilancia implementados al interior de los shopping centers como formas de, al mismo tiempo, desincentivar los robos y hacer que los visitantes se conformen a las prácticas sugeridas por los desarrolladores y consideradas normales al interior del mall.

Para algunos autores, mediante el control del espacio, las autoridades del mall han sido capaces, en forma efectiva, de eliminar las más abiertas prácticas de resis- 
tencia (Stahely \& Mitchell 2006; Davis 1990; Lofland 1998); o hacer que ciertos usuarios se conformen a las normas establecidas (Mugan \& Erkip, 2009); para otros, en tanto, las estrategias de control social, especialmente las destinadas a vigilar y controlar adolescentes, simplemente llevan a que los grupos vigilados elaboren prácticas de resistencia más complejas (Lewis 1989; Ortiz, 1994; Matthews et al., 2000).

\section{El mall como metáfora del neoliberalismo}

En el caso de Chile, dado que el mall surgió en pleno período de reestructuración económica en la década de los 80 , su valoración intelectual está íntimamente ligada a la valoración que se hace del neoliberalismo. Así, para quienes adherían a la dictadura y la economía liberal, la llegada de los malls fue el signo inequívoco del éxito económico del régimen y las transformaciones estructurales que promovió (Lavín, 1987), mientras que, para sus críticos, los centros comerciales aparecen como una afrenta para quienes, por su precariedad material, no pueden acceder al consumo. En la década siguiente, este debate sobre los malls y el rol del consumo se agudiza. Mientras Moulian (1997) ve en ellos el paradigma del consumo conspicuo, la "catedral del consumo" en el seno de una ciudadanía cada vez más individualista, autores como Tironi (1999) o Halpern (2002) terminan valorando las posibilidades de consumo de la población y la emergencia del "consumidor" como nuevo actor de la vida social de un país plegado a las dinámicas modernizadoras.

En línea con la renovación de los estudios urbanos y con la necesidad de combinar debate teórico con análisis empírico, el presente artículo buscó analizar cualitativa y etnográficamente las prácticas socioespaciales de adolescentes en el Mall Plaza Vespucio, así como las significaciones que ellos construyen sobre el centro comercial. Tomando parte de los debates teóricos internacionales, se deseaba establecer empíricamente si: (i) el centro comercial era para los adolescentes un espacio encerrado y aislado del resto de la ciudad; (ii) el centro comercial era un espacio hiper vigilado con altos niveles de control sobre las prácticas cotidianas de los visitantes; y (iii) si las prácticas desarrolladas en el centro comercial podían distinguirse de las llevadas a cabo por los adolescentes en otros espacios de socialización, como plazas, parques o calles comerciales tradicionales.

Con un sello cualitativo, el estudio de caso permitió el análisis de información primaria proveniente de un proyecto de investigación multimetodológico llevado a cabo entre los años 2008 y 2010. Para este artículo se utilizaron, específicamente, observación etnográfica no participante y entrevistas semiestructuradas.

En este estudio, la observación no participante comprendió anotaciones de campo obtenidas a partir de nueve meses de trabajo en terreno. Las observaciones fueron analizadas en reuniones semanales, a partir de las cuales fueron emergiendo, inductiva y deductivamente, categorías que guiaron los registros posteriores.

Una vez que se obtuvo una visión general de las prácticas adolescentes en el mall, se buscó obtener datos más directos mediante conversaciones con adolescen- 
tes que visitaban el lugar. En ese contexto, se entrevistó, en pequeños grupos, a 27 adolescentes autodefinidos como de "clase media".

Transcritas las entrevistas y la observación, se inició el proceso de codificación, el que implicó la generación de códigos abiertos que, con el correr del análisis, fueron convirtiéndose en códigos axiales que dieran cuenta de categorías y temáticas amplias. Al mismo tiempo que se codificaba, se fueron intercambiando notas y conversaciones que permitieron desarrollar conexiones entre la teoría y lo empíricamente descubierto (Glaser \& Strauss, 1967).

Los discursos de los entrevistados fueron entendidos como válidos pese a sus contradicciones. Tal aproximación ha sido descrita por la antropología cultural como perspectiva emic, la cual — contraria a una categorización sostenida exclusivamente en los criterios del investigador, o también llamada perspectiva etic- propone una investigación fundada en categorías y distinciones construidas, significadas y apropiadas por los propios actores (Harris, 1979).

Por último, se realizó una exhaustiva revisión de prensa escrita sobre el período de apertura del Mall Plaza Vespucio.

\section{Cuando la periferia se volvió subcentralidad: el Mall Plaza Vespucio de La Florida}

En el contexto de crítica y alabanza intelectual recién descrito, el análisis sobre el mall como fenómeno tangible e intangible estuvo localmente desatendido hasta mediados de los años 1990. Este panorama cambió años después, cuando, conscientes del impacto provocado por la inauguración de centros comerciales en sectores habitados por grupos medios y sectores populares, ${ }^{2}$ algunos autores ya reconocían sus virtudes concurrenciales e, incluso, lo consideraban un espacio público sustituto, resaltando impactos positivos y negativos en su entorno inmediato (Programa de Naciones Unidas para el Desarrollo [PNUD], 1998; Cáceres \& Farías, 1999).

Desde entonces ha persistido el intento de estudiar los malls como artefactos, con lógicas, prácticas y formas de operar características. Este análisis se ha desarrollado desde diversas ópticas, entre las que resaltan las adaptaciones locales según los contextos urbanos (Salcedo, 2003a, 2003b); las diferencias en prácticas de consumo entre niños ricos y pobres (Magendzo \& Bahamondes, 2005); la imagen ciudadana construida sobre el mall en tanto reflejo de la modernidad en otros sectores de la ciudad (Cáceres, Sabatini, Salcedo \& Blonda, 2006); el desarrollo de prácticas alternativas e ilegales en su interior en función de las amplias posibilidades de encuentro social (Stillerman, 2006); su importancia en el desarrollo de subcentros ur-

Cabe hacer notar que, en Chile, más del $85 \%$ de los ciudadanos se autoidentifica con la clase media, cuando en términos sociológicos ella no supera el 50-60\% de la población del país.

2 Específicamente: Plaza Vespucio en la comuna de La Florida (1990), Arauco Outlet Mall en la comuna de Maipú (1993), Plaza Oeste en el límite entre las comunas de Cerrillos y Maipú (1994), Mall del Centro en la comuna de Santiago (1996) y Plaza Tobalaba en la comuna de Puente Alto (1998). 
banos (Galetovic, Poduje \& Sanhueza, 2009), o la trasposición de prácticas sociales de otros espacios urbanos (Salcedo \& Stillerman, 2010).

La transición desde un gobierno militar a otro civil no sería la única novedad en el Santiago de 1990. A mediados de año, muy cerca de un cruce vial de tráfico concurrido, se inauguraría un edificio exclusivamente orientado al comercio minorista. Rompiendo la tendencia de localización de malls en el cono de alta renta (Cosmocentro Apumanque, Parque Arauco y Panorámico), la implantación del que más tarde conoceríamos como Mall Plaza Vespucio fue un acontecimiento metropolitano de primer orden.

Contorneado por una amplia playa de estacionamientos, morfológicamente el mall se presenta como cerrado y hermético hacia el exterior (Figura 1). Neomoderno antes que posmoderno, Plaza Vespucio disponía de un equipamiento cuya calidad era desconocida para grandes porciones de la ciudad. En contraste con la homogeneidad social típica de los centros comerciales del suburbio norteamericano (Cohen, 1996), el Mall Plaza Vespucio disponía del potencial para convertirse en una referencia para todos los grupos etarios, pero especialmente para los jóvenes.

FIgURA 1 | Mall Plaza Vespucio a inicios de los 90. Vista desde Vicuña Mackenna (Occidente)

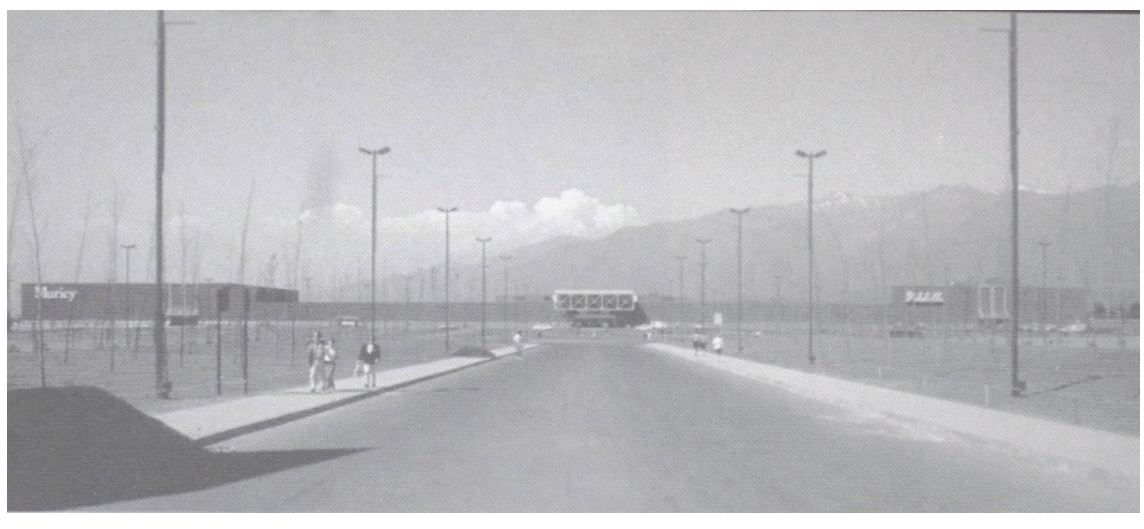

FUENTE CIUDAD Y ARQUITECTURA, 72 (1993), P. 51.

Con el paso de los años, y a pesar de ser desafiado por un competidor ubicado a menos de un kilómetro de distancia (Florida Center, inaugurado en el año 2003), Plaza Vespucio ha mantenido casi sin variaciones el liderazgo a escala metropolitana, tanto en términos de tamaño y número de visitantes, como de ingresos. De hecho, Plaza Vespucio sigue creciendo en áreas destinadas a tiendas y sumando nuevos espacios interiores, hasta llegar a tener hoy en día cerca de 180.000 metros cuadrados construidos. La cifra no deja de sorprender si lo comparamos con otros malls mundialmente conocidos por su magnitud, como el Mall of America de Estados Unidos y el West Edmonton Mall de Canadá (respectivamente 230.000 y 
500.000 metros cuadrados construidos, este último el centro comercial más grande del mundo hasta 2005). Por otro lado, las proyecciones anuales de visitas señalan que el año 2010, Plaza Vespucio recibiría 36 millones (Mall Plaza, s/f), una afluencia superior a los 28 millones de Mall of America, y ligeramente inferior a los 40 millones de West Edmonton Mall.

Volviendo a las razones del éxito del Mall Plaza Vespucio, es posible detectar cuatro elementos principales.

1) El mall está servido y conectado no solo a una red vial que lo hace accesible en automóvil, sino además a un expedito sistema de transporte público, proceso radicalizado por las extensiones del Metro hacia dicho sector (Línea 5 y Línea 4a) y por la construcción, colindante, de una estación intermodal de buses.

2) Los habitantes populares de la comuna de La Florida vieron la llegada del mall como la expresión material de la "modernidad" en una periferia, hasta entonces, casi totalmente dependiente del centro (Cáceres et al., 2006; Farías, 2009). Este juicio fue compartido por los formadores de opinión pública del momento, tal como se aprecia en un titular del diario La Tercera: "Con moderno centro comercial comuna de La Florida ingresa al año 2000” (3 de junio de 1990, p. 16).

3) El mall da cuenta de una importante aspiración identitaria de la clase media emergente que comenzaba a habitar la comuna de La Florida (Tironi, 1999; Rasse, Salcedo \& Pardo, 2009). Dicha dimensión queda claramente expresada en la propaganda inmobiliaria de los años 1990: "Los Jardines de Vespucio. Viva en el nuevo barrio alto sur oriente (...) A solo 20 minutos del centro de Santiago, centros comerciales de Providencia, y próximo [a] futuro Shopping La Florida" (Suplemento "Su Casa", La Tercera, junio 1990).

4) Plaza Vespucio se ha convertido, material y simbólicamente, en el principal ejemplo de la "democratización del consumo" vía masificación del crédito. Aquel fenómeno es ratificado en la distribución socioeconómica de los hogares que visitan el Mall Plaza Vespucio, cifras que hacia 2010 se repartían de la siguiente forma: $\mathrm{ABC} 1$ y C2, 34\%; C3, 45\%; y D, 21\% (Mall Plaza, s/f; nótese la desaparición del estrato $\mathrm{E}$, el cual, con presencia tangible en dicho centro comercial, es anulado como comprador en los sondeos realizados).

\section{Habitantes de la ciudad:}

los adolescentes en el mall

Las múltiples interacciones sociales ocurridas al interior del mall han sido una interesante dimensión de análisis, si se considera su naturaleza controlada y orientada al consumo. En este contexto, salta a la luz una categoría social especialmente incómoda para el centro comercial: los adolescentes (e.g. Abaza, 2001; Bermúdez, 2003, 2008; Voyce, 2006; Mugan \& Erkip, 2009). 
En los primeros estudios sobre adolescentes y malls aflora el merodear y "pasar el tiempo" (hang out) como términos cualificadores de las actuaciones de los adolescentes. Más que un consumidor, el joven estaría en el centro comercial por un interés casi exclusivamente social. En algunas de estas investigaciones, por ejemplo, se da cuenta de la gran cantidad de tiempo ocupado por los jóvenes en vagabundear por el shopping center versus el poco dinero gastado en consumo; o de las significaciones subjetivas del mall como un espacio "neutro" que, a pesar del constante hostigamiento de los sistemas de vigilancia, permite la construcción identitaria de un grupo etario que está social, sexual y económicamente excluido de la sociedad adulta (Anthony, 1985; Lewis, 1989). Así, para algunos autores, el centro comercial se transforma en instancia predilecta de socialización y satisfacción de necesidades emocionales adolescentes (Kim, Kim \& Kang, 2003); un lugar en que los jóvenes, pese a algunas quejas, no se sentirían mayormente discriminados (Mugan \& Erkip, 2009).

En ese entendido, se han destacado las disputas simbólicas entre adolescentes y adultos en el centro comercial. El flaneur inaceptable - como dirían Matthews et al. (2000) - sería un habitante arquetípicamente perturbador para los administradores del centro comercial, ya que su sola presencia lo convierte en sujeto amenazante para la atmósfera controlada del mall y sus consumidores reales o potenciales.

Con todo, rechazando los discursos intelectuales que minusvaloraban la actuación adolescente en el mall, Bermúdez (2008) señala que dicho espacio ha logrado posicionarse como una instancia de encuentro, de construcción y disputa identitaria. De este modo, yendo más allá de la mera constatación del ejercicio de la hegemonía, los centros comerciales no vienen a desplazar el parque o el barrio, sino a convertirse en un espacio urbano más de interacciones sociales donde se observa la diferencia y el conflicto.

Similar posición es observable en estudios desarrollados recientemente en Santiago de Chile (Salcedo \& Stillerman, 2010; Stillerman, Salcedo, Philips \& Covarrubias, 2010), los que pusieron atención en la heterogeneidad social circundante en dos shopping malls de la comuna de La Florida (Plaza Vespucio y Florida Center). De sus conclusiones extraemos dos puntos importantes: primero, la idea de que es posible describir las prácticas socioespaciales de los adolescentes como "transposiciones" de otras prácticas provenientes de espacios públicos tradicionales (Salcedo \& Stillerman, 2011); y segundo, el reconocimiento del centro comercial en tanto instancia de construcción identitaria de jóvenes autoadscritos a la clase media y la legitimación subjetiva de su uso mediante las prácticas de consumo (Stillerman et al., 2011).

$\mathrm{Al}$ igual que para otras latitudes, los estudios realizados sobre experiencias ocurridas en Chile están marcados por las transformaciones culturales que los jóvenes han experimentado en las últimas décadas. Específicamente, pensamos en su retirada de la esfera pública y el predominio de referentes más personales que colectivos en su construcción identitaria (Programa de Naciones Unidas para el Desarrollo [PNUD]/ Instituto Nacional de la Juventud [INJUV], 2003; Marín, 2008). 


\section{Prácticas adolescentes en el mall y sus alrededores: disrupción, represión y eterno retorno}

Las prácticas adolescentes que tienen lugar en el Mall Plaza Vespucio y sus alrededores son de dos tipos: de consumo y de interacción social.

La concepción del mall en tanto escenario de sociabilidad adolescente implica que las prácticas de consumo tengan una relevancia mínima en la descripción que los jóvenes hacen de sus rutinas en el centro comercial. El consumo como práctica depende, entonces, totalmente de las circunstancias (familiares o sociales) que causaron la concurrencia al mall: si son los padres quienes empujaron al adolescente a recorrer Plaza Vespucio, es porque específicamente se va de shopping. Más aún, el ser observado con los padres en el mall en situaciones ajenas al consumo es mal visto por los adolescentes, hombres y mujeres:

Vai caminando y es como... “ay, está con los papás, no sale sola”, y es como “oh...”. Y además que, no sé, por ejemplo uno ve a alguien y tu mamá empieza como "hola, como estái", y la cuestión, y "llévalo a la casa a tomar té”. (Sol, 14 años).

Por otro lado, si el motor de la visita fue de índole social, el consumo es una de las tantas acciones realizadas al interior del mall, un accesorio al vagabundeo o la socialización. Así, mientras se está con amigos, es posible consumir alimentos o bebidas o utilizar algunas instalaciones, como el bowling o los juegos electrónicos. Con todo, muchos adolescentes reconocen que el consumir algo se convierte en una forma de legitimar su presencia en el centro comercial: implica una aceptación tácita de las reglas y una forma de convertirse en "cliente" (Chin, 2001; Stillerman et al., 2010).

Este deseo de legitimar la propia presencia tiene que ver con la vigencia en el mall de un aparato punitivo que busca evitar la aparición de conductas poco compatibles con su función comercial. Al respecto, nuestras observaciones nos llevan a establecer que, tal como ha sido descrito en otros trabajos, tanto nacionales como extranjeros (e.g. Stillerman \& Salcedo, 2011; Mugan \& Erkip, 2009), la actitud de los vigilantes privados al interior del centro comercial dista mucho de ser minuciosa e inhibitoria. Por más que, reconocidamente, un shopping center promueva el control exhaustivo de las prácticas socioespaciales generadas dentro de sus márgenes, durante el trabajo de campo pudimos notar la presencia impertérrita de los guardias ante hechos claramente repudiables para los administradores del mall, como comercio informal, "carretes" juveniles, mendicidad, e inclusive paseos caninos. Ello nos hace reflexionar sobre la capacidad real de negociación que tienen los usuarios del mall, la que, por un lado, implica — consciente o inconscientementeaceptar ciertas reglas (como la necesidad de comprar); y por otro, también implica la repetición constante de prácticas indeseables que, con el correr del tiempo, dejan de ser perseguidas por la autoridad.

En cualquier caso, son las interacciones sociales, como un tipo de acción colectiva, las que explican de manera mayoritaria la asistencia adolescente al mall. Inde- 
pendientemente de la intensidad de las relaciones producidas en el shopping center, para nuestros fines resulta interesante reflexionar sobre la capacidad interaccional del mall. Para ello, necesariamente debemos atender a la dimensión espacial, pues dichas interacciones no se producen con igual intensidad ni de igual forma en todos los espacios del mall. Así, reconocemos dos grandes áreas geográficas, cualitativamente importantes, en las que las interacciones sociales adolescentes se desarrollan y en las que se establecen relaciones diferenciales con los sistemas de control: (i) Las Terrazas, de Plaza Vespucio/el patio de comidas; y (ii) el sector Aires.

Las Terrazas, de Plaza Vespucio, es una estructura abierta, de dos plantas, inaugurada el año 2005. Con sus improvisados escaños (jardineras, escaleras e inclusive el suelo) y sus pasarelas invita a la permanencia constante y significativa de los usuarios, todo ello amenizado por los restoranes, salas de teatro, de cine y múltiples posibilidades de acceso que presenta (desde el exterior, por la calle Froilán Roa y los estacionamientos; desde el mall, por los pasillos en el primer nivel y por el patio de comidas en el segundo piso).

Podemos pensar que la opción de diseño de este espacio no fue baladí. Mamparas, amplios ventanales y puertas no hacen más que intentar convencer a los usuarios de que, al menos en apariencia, se ha dejado atrás el mall convencional y enclaustrado, accediendo a lo más parecido a una plaza pública que Plaza Vespucio puede ofrecer. En este contexto, tanto nuestras observaciones como nuestras entrevistas nos llevan a pensar que las dicotomías público/privado, adentro/afuera, se hacen difusas y complejas de sostener por los visitantes adolescentes, desapareciendo las certezas del usuario respecto de las normas del mall y la forma en que ellas se harán cumplir.

Como que de repente igual uno va a otro mall y de repente los jóvenes quieren fumarse un cigarro y como que tienen que salir y... En cambio por aquí [por Las Terrazas] salís al patio y después entrái al tiro. (Alexander, 16 años).

De esta manera, la distinción entre la porción interna y externa del food court (patio de comidas y Las Terrazas) es puramente metodológica, pues la primera no es comprensible sin la segunda, dado que las prácticas adolescentes ahí desarrolladas trascienden el consumo de alimentos.

Aires, inaugurado a fines del año 2008, es una de las últimas grandes apuestas de Mall Plaza Vespucio para atraer a aquellos estratos medio-altos mayoritariamente ausentes del centro comercial. Con una inversión de 28 millones de dólares, Aires incluye tiendas exclusivas nunca antes vistas en la comuna de La Florida, así como una opción de diseño radicalmente diferente al del resto del centro comercial, en la que la incorporación de luz natural, musicalización, jardines y cascadas de agua, enfatizan mucho más el lujo que la funcionalidad. Desafiando un historial casi perfecto de aciertos, al ver el vacío total de la segunda planta de Aires, podríamos aventurar que el plan inicial de los desarrolladores no ha sido, hasta ahora, del todo exitoso. 
A pesar de lo anterior, Aires ha logrado captar la atención de los adolescentes. Es este espacio, aprovechado por su escasa afluencia de público, el preferido por los adolescentes para sus rituales de cortejo, citas y romances. Es también un lugar confortable para grupos pequeños que se reúnen a conversar.

Cabe hacer notar, en cualquier caso, que la desidia de los guardias, que es bastante típica del sector Las Terrazas/patio de comidas, se altera sustantivamente en el Aires, lo que genera una percepción radicalmente diferente respecto al control espacial por parte de los usuarios adolescentes, tal como se aprecia en las siguientes citas:

[En Aires] no podemos gritar y cuestiones... [En cambio] aquí en Las Terrazas uno se tira al suelo si quiere, te tirái al suelo, bailái, hacís lo que querái. (Carla, 16 años)

Yo soy jefa de team [grupo de amigos de la tribu urbana pokemones], entonces a veces igual cuesta, porque los chiquillos se ponen muy desordenados. En Aires empiezan a tirar "challa", cucharas, lo que sea, entonces la gente se molesta y uno tiene que estar ahí diciéndoles a los chiquillos que no hagan eso pa' que no nos echen, pa' no tener ata'os. (Laura, 15 años).

Esta diferencia de percepción es el reflejo de una realidad más profunda. Aunque sabemos que el ejercicio represivo está presente siempre y en cada momento, podemos ver que los dispositivos de seguridad de Plaza Vespucio arremeten y se hacen visibles en distinto grado y frente a distintas acciones, según sea el recinto. El espacio más íntimo y lujoso de Aires es cuidado en sus detalles y, por ende, las prácticas son más restringidas; por su parte, la densidad urbana y la apertura hacia el exterior de Las Terrazas/patio de comidas imposibilitan el control total del espacio, eligiéndose reprimir solo algunas prácticas y dejando el resto en la invisibilidad del anonimato denso.

De cualquier modo, sabemos que existen algunas prácticas que son reprimidas en todo contexto y lugar: "poncear" (besos y caricias fogosas), "carretear" (juerga sin término), o patinar en skate. Ello lleva a que los adolescentes solo las desarrollen luego de una evaluación minuciosa del entorno físico-social circundante y de la posibilidad/imposibilidad de ser reprimidos por los guardias. Más aún, aquellos que desarrollan estas actividades van formando una suerte de "cultura nómada" en el mall, con continuos desplazamientos y cambios en sus lugares de reunión e interacción.

[Hay que] estar escondido de los guardias... Cuando llegan los guardias a echarnos [de Las Terrazas] nos vamos pa' Aires; después, cuando nos echan de Aires, nos vamos al [paseo El] Cabildo, y así. (José, 17 años).

En la serie de prácticas adolescentes que se enfrentan al ejercicio disciplinario del mall, podemos pensar su progresión desde la relación constante entre el control y la resistencia en los términos en los que esta dicotomía fue planteada por De Certeau (1984); es decir, como una disputa de corto alcance que modifica los usos y sentidos iniciales que los productores del espacio imaginaron en un comienzo. Si el food court fue diseñado para comer permaneciendo el menor tiempo posible, 
los adolescentes utilizan el mobiliario para pasar largas horas "carreteando"; si los sillones de Aires están dispuestos para acomodar al cliente de sus costosas tiendas, los adolescentes se acarician con pasión en ellos.

Tal como señalaba Lewis dos décadas atrás (1989), la presión del mall por controlar dichas acciones y prácticas es totalmente soportable por los adolescentes, ya que no implica agresiones físicas graves ni prohibiciones de acceso permanentes. Ello va generando un juego de persecución constante entre guardias y adolescentes. Aún más, su rutinización ha permitido el conocimiento mutuo entre celadores y celados, haciendo factible el eterno retorno al mall.

Los guardias nos "cachan" [conocen] más porque, claro, venimos todos los fines de semana, entonces ya nos ubican. (Conversación informal, adolescente aproximadamente 16 años)

\section{Significaciones adolescentes sobre Plaza Vespucio: ¿el mall como plaza pública?}

Comencemos por mencionar la situación observada entre algunos jóvenes que, teniendo otros centros comerciales más próximos a sus casas o colegios, prefieren concurrir a Plaza Vespucio. Muchos de los entrevistados provienen de comunas distantes de La Florida (e.g., Pudahuel, Maipú, Santiago), mientras otros, residiendo en otros municipios adyacentes, como Puente Alto y Macul, no se plantean la posibilidad de ir a otros shopping centers del suroriente de Santiago (Florida Center y Plaza Tobalaba).

El [Mall Plaza] Tobalaba queda más cerca de mi casa... [Pero] es como que hay menos gente y es fome... Llegái y está lleno de viejitos. (Alberto, 15 años).

Como que el [mall] del 14 es el más grande de todos, [pero] el Florida Center es para andar en familia igual y es más caro. (Sol, 14 años).

No tenemos idea de las razones por las que venimos [a Plaza Vespucio]; es que el team nació acá y [aunque] uno trata de sacarlos..., porque hemos ido al paseo Quilín, al Florida Center, pero siempre terminamos acá. (Laura, 15 años).

Las citas anteriores nos permiten extraer importantes conclusiones respecto de los discursos legitimadores que los adolescentes fabrican para explicar su presencia en el Mall Plaza Vespucio.

a) La accesibilidad, como aspecto material, claramente posee una relevancia secundaria, toda vez que muchos jóvenes prefieren asistir a este mall aun teniendo otro más próximo.

b) Centralmente, el discurso legitimador es de carácter simbólico: de los adolescentes existe una "primacía subjetiva" de "el 14" respecto de otros centros comerciales. Esta centralidad está dada ya sea por su tamaño, la cantidad de amenidades presentes en él, o la atmósfera provocada por la gran cantidad y diversidad de personas que lo visitan. 
c) Además, existe otro discurso legitimador de la propia presencia en el Plaza Vespucio que se liga a razones de tipo económico: "los otros malls son más caros". Para los adolescentes entrevistados, Plaza Vespucio representa una alternativa "más a su alcance" que otros espacios, como Florida Center o los malls del barrio alto de la ciudad de Santiago.

Sin embargo, esta justificación económica tiene menos que ver con un análisis comparado de los precios de determinados productos en distintos centros comerciales, que con una imagen de "mall de clase media" que se ha forjado el Plaza Vespucio, imagen que guarda relación tanto con la autopercepción de los jóvenes, como con sus pautas y formas de consumo. Los jóvenes entrevistados, tal como aparece en otro estudio (Stillerman et al., 2010), valorizan la austeridad y "racionalidad" del consumo de clase media, en contraste con la "irracionalidad" y muchas veces los excesos en el consumo de jóvenes de estratos tanto altos como populares.

Este carácter de clase media del Mall Plaza Vespucio no implica que entre su concurrencia no se adviertan personas de estratos sociales altos o bajos. Esta diversidad de usuarios es valorada positivamente por los adolescentes entrevistados, pues refleja una pluralidad social no apreciable en otros shopping centers. Tal juicio es especialmente referido a los centros comerciales del barrio alto (e.g., Parque Arauco, Alto Las Condes), en los que, según las construcciones imaginarias de nuestros entrevistados, la diversidad social es escasa, presentándose como espacios estandarizados, ritualizados y carentes de toda contingencia. En todo caso, se debe recalcar el carácter "imaginario" de este discurso, pues muy pocos entrevistados afirmaron haber asistido, al menos, con algún grado de regularidad a estos malls del barrio alto.

Allá es como otro ambiente, porque allá son todos más lais [de mayor nivel socioeconómico]. (Claudia, 15 años).

Al considerar la concurrencia pluriclasista del Mall Plaza Vespucio, se hace posible comprender el discurso juvenil respecto de la apropiación de ciertos espacios: la identidad de lugar se construye mediante la disputa y el conflicto con otros jóvenes - sean estos de otras clases sociales o pertenecientes a alguna tribu urbana-, así como, en muchos casos, con los guardias y otras autoridades adultas en el mall. Como dijimos, la presencia del otro es valorada (discursivamente), pues genera diversidad, pero al mismo tiempo puede llegar a constituir una amenaza y una dificultad para llevar a cabo la apropiación simbólica de ciertos territorios.

La existencia de grupos amenazantes, cuyo origen social es adscrito a barrios populares, y que estarían siempre dispuestos a actuar violentamente, fue presentada frecuentemente como uno de los problemas que tendría el Mall Plaza Vespucio. La colonización del centro comercial por parte de grupos de "flaites" (nombre peyorativo y estigmatizador dado a los adolescentes del mundo popular con estética estilo reggaeton $^{3}$ ) vendría a tensionar una instancia de sociabilidad aparentemente segura.

3 Definida por uso de ropa deportiva de marcas reputadas — Adidas, Puma, Nike-, zapatillas ostentosas, bling-bling (joyas colgadas al cuello) y corte de pelo estilo "sopaipilla"; esto es, rapado totalmente en las sienes y la nuca, mientras en la zona occipital el cabello se mantiene ligeramente más largo, con un meticuloso arreglo. 
[Una vez] el team de nosotros le pegó al team de ellos (...) y ellos tenían como lazos con los flaites; entonces después nos querían pegar y tuvimos que dejar un tiempo de venir al mall, acá a Las Terrazas. (Ricardo, 16 años).

Este "flaite" es tolerado, y su presencia no es, por lo habitual, lo suficientemente amenazadora como para desincentivar o evitar en forma permanente la propia visita, dado que la interacción con ellos se produce en un ambiente seguro y controlado. Así, al tiempo que el guardia siempre es visto en términos negativos como el "represor", inconscientemente su presencia y la de otros mecanismos de control espacial otorgan el marco de seguridad necesario para el encuentro pluriclasista. Por tanto, aunque haya "flaites" por doquier, Mall Plaza Vespucio permite idealmente la interacción y reconocimiento con los otros en una atmósfera dinámica, algo caótica, dispuesta a aceptar una multiplicidad de agentes, pero controlada y segura.

Ahora bien, el mix social, el control del espacio, o la presencia de otros consumidores, son solo algunos de los aspectos que los adolescentes deben tener en cuenta en el desarrollo de sus tácticas de apropiación del lugar (De Certeau, 1984). Dichas tácticas requieren conocimiento cabal del espacio y sus reglas, repetición de prácticas y estrategias en un contexto de ensayo y error; en fin, requieren una inversión de tiempo e inteligencia colectiva. Esta inversión es la que nos permite pensar en la generación de profundos lazos emocionales con un espacio que ha sido fuertemente significado durante sus historias de vida. Como ellos mismos señalan, "el mall siempre estuvo alli", y probablemente es lo que justifica también la no visita a otros malls, en los que tendrían que re-aprender códigos de apropiación y estarían expuestos a contingencias inciertas.

\section{El mall, la calle y la plaza en el discurso y prácticas adolescentes}

La naturalización del mall por parte de sus usuarios (Stillerman \& Salcedo, 2010) implica que la pertinencia de ese escenario para el establecimiento de las relaciones sociales no es cuestionada, lo que trae consigo una pregunta crucial: ¿̇erán abandonados por los adolescentes espacios como la calle o la plaza? ¿Terminará el mall reemplazando a los denominados "espacios públicos", como sostienen sus críticos?

Para responder estas preguntas debemos analizar la comparación calle/plazas versus mall que hacen los adolescentes entrevistados, así como también los distintos lugares y la forma en que ellos los van habitando cotidianamente.

Entre los principales aspectos del mall rescatados por los adolescentes, están las posibilidades que este espacio ofrece para establecer sus interacciones sociales en contextos de mayor seguridad que otros espacios de la ciudad.

Acá es mejor porque el [paseo El] Cabildo es más peligroso, porque siempre nos quieren como... asaltar (...) igual que en otros lados, como el Parque La Bandera en San Ramón. (Daniel, 16 años). 
La seguridad, no obstante, es para los adolescentes una variable que posee dimensiones positivas y negativas: aunque los dispositivos del mall reducen considerablemente, aunque no eliminan, los riesgos de ser victimizado, al mismo tiempo limitan la espontaneidad de las interacciones mediante el control de las prácticas socioespaciales. No por nada, los "otros significativos" para los adolescentes que entrevistamos son los "flaites" y los guardias: los primeros, como potenciales agentes victimizadores; y los segundos, como actuales agentes represores.

Sin embargo, a pesar de concentrar riesgos en sus usos, los espacios como calles y plazas no son desechados por completo, en la medida en que otorgan grados de libertad mayores que el mall. Plazas de barrios y calles no desaparecen del discurso adolescente, sobre todo si se trata de interactuar en circunstancias u horarios en los que el mall no lo permite. El centro comercial es visto como un espacio más para ocupar, con sus propias reglas de apropiación, sus ventajas y sus problemas. Es un escenario que, siguiendo a García Canclini (1999), podría interpretarse desde el sentido mismo de la vida urbana, a saber, como un lugar para "habitar" y "ser imaginado", que emerge de aquellas comunicaciones múltiples y heterogéneas propiciadas por los habitantes de la ciudad. Consecuentemente, siendo un tipo de "espacio habitado", según lo entendido por De Certau (1984), el mall nacería de prácticas y operaciones en pugna que lo forman, lo determinan y lo sitúan, cuestión que permite a los adolescentes, por ejemplo, establecer relaciones sistémicas entre este espacio aparentemente privado y los espacios públicos circundantes. Para los adolescentes entrevistados, y para muchos otros que observamos, el mall no es un lugar cerrado y desligado de su entorno, sino más bien uno entre varios espacios que conforman un "lugar" en el que ellos van desarrollando sus relaciones sociales y simbólicas. Este lugar, denominado por nuestros entrevistados como "Paradero 14" o simplemente "el 14", incluye espacios privados como el mall, espacios comerciales tradicionales, paseos peatonales, plazas y áreas verdes, etcétera. "Siempre vamos a terminar en el 14" (Laura, 15 años), afirmó una informante que, siendo entrevistada al interior del mall, jamás comprendió los límites físicos y jurídicos que separaban ese lugar, originalmente pensado como un emprendimiento privado con fines de consumo, de su entorno.

Esta relación sistémica y de complementariedad se aprecia particularmente bien al analizar los movimientos y migraciones adolescentes que ocurren entre el Mall Plaza Vespucio y el paseo (calle) El Cabildo, espacio comercial tradicional y abierto del paradero 14 (Figura 2), y las significaciones que los adolescentes hacen de este paseo y su encuentro con el centro comercial.

Con su feria artesanal - lugar predilecto para el consumo cultural de algunos adolescentes-, vendedores ambulantes, supermercados en sus contornos, gitanas, áreas verdes un tanto descuidadas y la casona municipal de La Florida, el paseo peatonal El Cabildo es el espacio público con mayor intensidad en el uso adolescente en las afueras del mall. En tanto punto de encuentro de quienes vienen de distintas latitudes y refugio momentáneo de los expulsados del centro comercial, el paseo El Cabildo y el Mall Plaza Vespucio funcionan integradamente como un gran espacio de interacciones sociales para los jóvenes; espacio en el que se produce el encuentro 
—y, hasta cierto punto, la confusión — entre el afuera/adentro, público/privado, tradicional/moderno, local/global.

Más que competir por la atención adolescente, ambos escenarios (Plaza Vespucio/El Cabildo) generan un virtuoso circuito de sociabilidad para los jóvenes que visitan el lugar, construyendo en la práctica lo que en su lenguaje cotidiano y su discurso los adolescentes denominan "el 14". Así, a pesar de su aparente impenetrabilidad, el mall aparece como un espacio poroso del que los visitantes entran y salen a voluntad, desplazándose hacia espacios aledaños de naturaleza jurídica y funcional radicalmente diferentes. Más aún, en nuestras observaciones pudimos apreciar que el mall es incluso utilizado como lugar de tránsito para desplazarse entre dos puntos.

FIGURA 2 | Plano paradero 14 La Florida. Lugares frecuentados por adolescentes

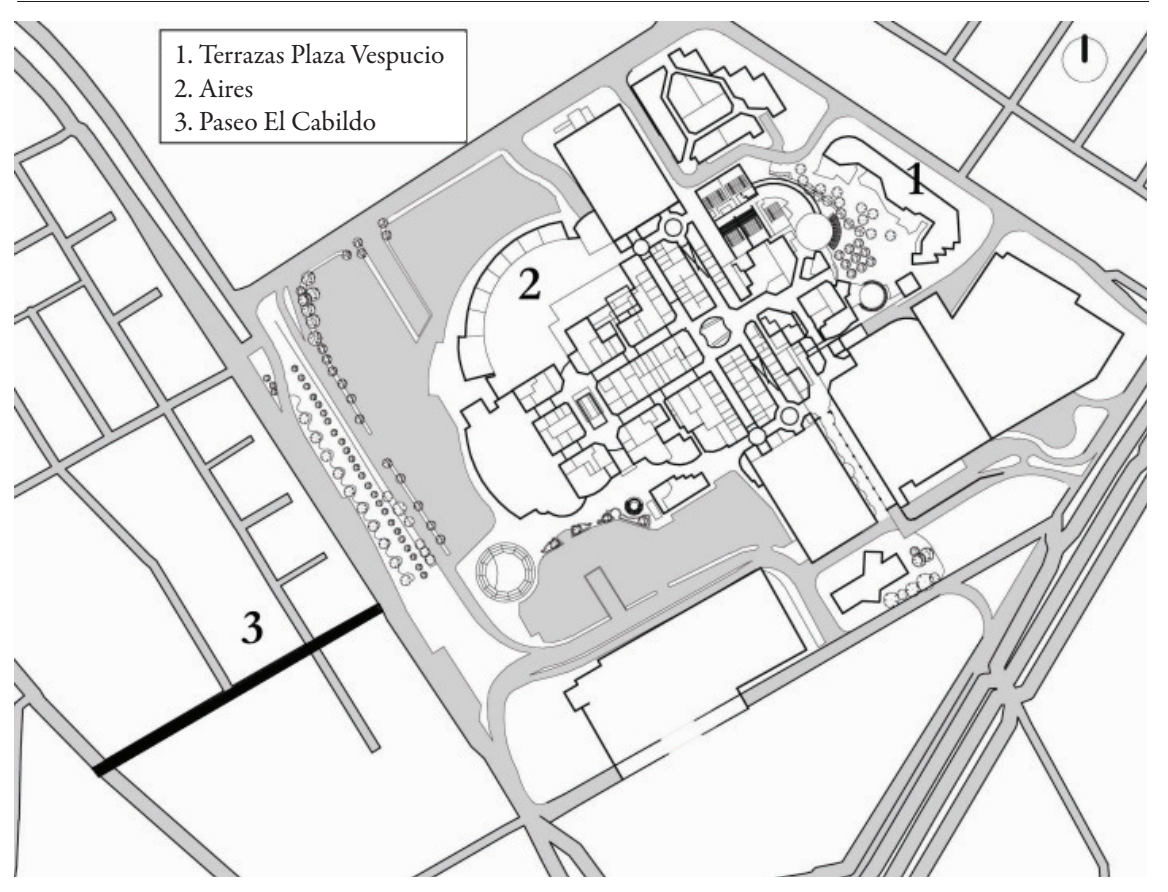

fuente Elaborado por Liliana de Simone. Escala 1:5000.

\section{Reflexiones finales: ¿nuevas formas de practicar la ciudad?}

En el éxito de Plaza Vespucio se conjugan factores urbanos y económicos, como la existencia de un sistema de transporte público expedito, la búsqueda identitaria de una comuna que progresivamente recibía a grupos medios y la masificación del crédito entre emergentes capas sociales. Dichas categorías nos hablan de variables 
coyunturales propicias para el desarrollo fructífero de un centro comercial, mas no refieren a las disposiciones socioculturales que estructuraron las valoraciones adolescentes sobre el Mall Plaza Vespucio. Sobre tal punto centraremos nuestras reflexiones finales, las que buscan sostener teóricamente lo que observamos en terreno. A saber:

a) Una cierta continuidad en el discurso y en las prácticas adolescentes entre el shopping center y los espacios aledaños a este, como plazas o calles comerciales tradicionales, continuidad que convierte el paradero 14 en un "lugar antropológico" (Auge, 2004), un sistema comercial y de espacio público con una identidad propia y que claramente excede la identidad del Mall Plaza Vespucio.

b) Una determinada forma de habitar el mall por parte de los adolescentes, y una adecuación a las condiciones de posibilidad que ofrece el lugar. Esta forma de habitar incluye la naturalización del espacio, la transposición de prácticas propias de otros lugares, la acomodación de prácticas, la resistencia activa o pasiva frente a las restricciones propuestas por la autoridad, y el nomadismo continuo como forma de permanencia.

Extrapolando al análisis urbano el concepto de habitus desarrollado por Pierre Bourdieu (1990), podemos pensar la constitución de un campo urbano a partir de la relación entre distintos agentes que, teniendo distintas dotaciones de capital económico, cultural, político y simbólico, han dibujado fracciones de la ciudad y, con ello, determinadas clases de conductas y percepciones sobre los espacios urbanos. Considerando este marco conceptual, podemos entender el desenvolvimiento de las prácticas socioespaciales adolescentes (en o sobre el mall), no tanto como prácticas "racionales" (como los teóricos liberales o los desarrolladores de malls pudieran pensar), sino más bien como prácticas razonables o de sentido común.

Así, mientras el mall ofrecería "condiciones de posibilidad", dadas por ciertos discursos y prácticas hegemónicas generadas desde los creadores y administraciones del espacio, los adolescentes actuarían en los márgenes, alterando significados, evadiendo controles o generando resistencias específicas, mas nunca generando proyectos alternativos de resistencia frontal o abandonando las significaciones generales que la población posee sobre el shopping. Esta visión nos permite, conceptualmente al menos, abandonar la dicotomía planteada por la literatura entre estructura (prácticas absolutamente condicionadas por los productores) y agencia (libre albedrío espacial total).

Dado que las "dualidades poder/resistencia al poder" o "productores/usuarios del espacio" se presentan en todos los espacios, incluidos los espacios públicos (Salcedo, 2002), no es curioso que los adolescentes conciban el mall como una "parcialidad urbana" que se conjuga con otros espacios de la ciudad, no cuestionando su uso bajo oposiciones teóricas del tipo espacio público/espacio privado, o calles y plazas/ centros comerciales. El centro comercial se engarza material y simbólicamente entre 
los paisajes de la ciudad: no compite con el barrio, la plaza o la escuela, sino que más bien los complementa, formando un circuito espacial de distintas interacciones.

Siendo parte de un habitus, las prácticas adolescentes en Mall Plaza Vespucio responden a determinantes socio-históricas, donde el mall se ha constituido como instancia nueva de sociabilidad de la juventud; y en el caso del Plaza Vespucio, como un espacio de sociabilidad e identificación de la clase media. Su naturalización ha llevado a desarrollar prácticas sociales, ciertamente observables en otros espacios urbanos, dentro de sus fronteras. En palabras de Salcedo y Stillerman (2010) sería una "transposición". Esta transposición, en cualquier caso, da cuenta de las condicionantes sociales y la historicidad del centro comercial, pues es claro que este "traer" prácticas desde los espacios públicos tradicionales nunca deja de considerar que ellas han de desarrollarse dentro del habitus propio de la "ciudad de los negocios" (Gorelik, 2004 [1994]). Dentro de este habitus, el control de las acciones emprendidas en el mall está, de manera paradójica, igualmente interiorizado por los adolescentes. Así, el hostigamiento es incluido dentro del total de fenómenos con los cuales se debe lidiar en la experiencia del mall.

Anteriormente habíamos aclarado la utilización del concepto "resistencia" acuñado por De Certeau (1984) con el fin de despojarlo de toda posible asociación con la imposición de proyectos políticos alternativos y contra-hegemónicos. Sumado a eso, diremos que en la constante negociación de lógicas e intereses nacidos de las disrupciones adolescentes, resaltan las dimensiones sociales propias de la producción del espacio (Lefebvre, 1991). Allí, en el "espacio vivido", los aspectos simbólicos que hegemónicamente dominan el espacio ("espacio concebido") son cambiados constantemente por los agentes urbanos, en virtud de la capacidad transformadora de la praxis de los usuarios; en nuestro caso, de los adolescentes.

En el desarrollo de la investigación, la continuidad espacial del vagabundeo adolescente (que los hace transitar en un eterno retorno entre centro comercial y espacios circundantes) nos obligó a expandir nuestra mirada fuera del mall, cuestionando severamente el carácter de "isla" o "negación de lo urbano" que le atribuye la literatura. El paseo El Cabildo fue especialmente relevante en su carácter de contenedor de las prácticas excluyentes empleadas por Plaza Vespucio hacia los jóvenes; destierros que, por cierto, no dejaban de ser momentáneos. Aunque reconocemos las particularidades únicas de este paseo peatonal en cuanto a localización, acceso e instalación en los bordes de uno de los centros comerciales más exitosos de la capital, las lógicas de El Cabildo abren la reflexión sobre la relación establecida entre un espacio público de carácter tradicional y su par privatizado.

En este caso, al contrario de lo que plantearía Sarlo en Escenas de la cultura postmoderna (1994), la dinámica urbana que se observa en el paradero 14 no solo no se ve obstaculizada por la presencia del mall, sino que, por el contrario, depende en gran medida del polo de atracción constituido por este. Así, al observar las prácticas cotidianas de los usuarios, podemos advertir que la instalación del mall no provocó la total anulación del espacio público circundante, sino más bien su dinamización y ampliación. 
La importancia social y política de la centralidad urbana ha sido ya largamente defendida por Lefebvre (1996), para quien —en un período de total urbanización de la sociedad - "el derecho a la ciudad" pasa por una recomposición de las centralidades donde la ciudad en su "valor de uso" (esto es la "ciudad como obra", opuesta a la "ciudad como producto") tenga una realización práctica. Aunque la transformación del paradero 14 no se explica en absoluto por el proceso revolucionario anhelado por Lefebvre, sí pone de manifiesto una profunda modificación tanto de las jerarquías urbanas dentro de Santiago (especialmente en la oferta de bienes y servicios) como de la naturaleza de la vida urbana que allí se desarrolla. En este contexto, reconocemos que uno de los mayores logros del "Mall del 14" fue llevar la intensidad de lo público, hasta entonces radicada solo en las áreas centrales de la ciudad, a una periferia mal equipada y con carencia casi total de vida urbana pública.

Lo anterior nos muestra que la interdependencia y continuidad entre ciudad y espacio privado (a pesar de todos los conflictos o roces producidos) puede ser entendida como una forma en que la ciudad —y, más específicamente, lo que la literatura denomina "espacios públicos" - asegura su supervivencia, su enriquecimiento y, más aún, su complejización en el contexto de la modernidad avanzada. Más que competencia, cuando el mall está dispuesto a abrir sus bordes (tal como lo ha hecho Plaza Vespucio), la ciudad y sus espacios públicos parecen buenos vecinos y un gran complemento frente a una oferta espacial que, si bien es amplia, no es total.

Por tanto, para nuestros informantes, los espacios privatizados y públicos no compiten en las mismas dimensiones, ya que, en su conjunción, conforman todas aquellas instancias de sociabilidad significativas, cada una con sus ventajas y desventajas. Mientras el uso de calles y plazas trae riesgos asociados a la victimización que se pudiera sufrir, el centro comercial garantiza una interacción social donde peligros de ese tipo están altamente reducidos. Por el contrario, mientras en su afán por la seguridad el mall puede reprimir determinadas prácticas socioespaciales de adolescentes, llegando, incluso, a vetar su presencia provisoriamente, los espacios públicos tradicionales se presentan libres y abiertos a albergarlas.

Consecuentemente, "libertad" y "seguridad" son dos categorías dicotómicas de especial relevancia en la comprensión discursiva de los adolescentes sobre los espacios de sociabilidad, toda vez que el desarrollo de una involucra el aplacamiento de la otra. Sin embargo, las diferencias que pudieran incidir en la valoración disímil del mall y el espacio público tradicional, resultantes del análisis de la dialéctica "libertad/seguridad", son resueltas por la experiencia de la diversidad practicable tanto en Mall Plaza Vespucio como en el paseo El Cabildo. Sobre estos marcos experienciales opera el nuevo habitus urbano de los adolescentes: haciendo posible la consideración como "público" de un espacio privado y sometiendo (o minusvalorando) el control de ciertas prácticas en función de la experiencia de diversidad y tolerancia frente a muchas conductas vividas en el mall. Todo eso, en un contexto en el que el espacio público no desaparece del horizonte juvenil y sigue recibiendo una pluralidad de actores, usos y prácticas, como queriendo desestimar a todos aquellos que han declarado, apresuradamente, su decadencia. IEURE 


\section{Referencias bibliográficas}

Abaza, M. (2001). Shopping malls, consumer culture and the reshaping of public space in Egypt. Theory Culture Society, 18(5): 97-122. doi: 10.1177/02632760122051986

Amendola, G. (2000). La ciudad postmoderna. Madrid: Celeste.

Anthony, K. (1985). The shopping mall: A teenage hangout. Adolescence, 20(78), 307-312. Disponible en https://www.ideals.illinois.edu/bitstream/handle/2142/25502/2324_001.pdf? sequence=2

Augé, M. (2004). Los "no lugares", espacios del anonimato. Una antropología de la sobremodernidad. Barcelona: Gedisa.

Bermúdez, E. (2003). 'Mall', consumo cultural y representaciones de identidad juvenil. En D. Mato (Ed.), Maracaibo. Políticas de identidades y diferencias sociales en tiempos de globalización (pp. 173 - 192). Caracas: Facultad de Ciencias Económicas y Sociales, Universidad Central de Venezuela (FACES - UCV).

Bermúdez, E. (2008). Roqueros y roqueras, pavitos y pavitas, skaters, lesbianas y gays. El papel del consumo cultural en la construcción de representaciones de identidades juveniles. Revista Latinoamericana de Ciencias Sociales, Niñez y Juventud, 6(2), 615-666. Disponible en http://redalyc.uaemex.mx/src/inicio/ArtPdfRed.jsp?iCve=77360206

Bourdieu, P. (1990). The logic of practice. California: Stanford University Press.

Cáceres, G. \& Farías, L. (1999). El espacio urbano. Efectos de las grandes superficies comerciales en el Santiago de la modernización ininterrumpida. Ambiente y Desarrollo 15(4), 36-41. doi: $10.3900 /$ fpj.7.6.380.s

Cáceres, G., Sabatini, F., Salcedo, R. \& Blonda, L. (2006). Malls en Santiago: Luces y claroscuros. ARQ, 62, 49-50. doi: 10.4067/S0717-69962006000100009

Chin, E. (2001). Purchasing power: Black kids and American consumer culture. Minneapolis: University of Minnesota Press.

Ciccolella, P. (1999). Globalización y dualización en la región metropolitana de Buenos Aires. EURE, 25(76), 5-28. doi: 10.4067/S0250-71611999007600001

Cohen, E. (1996). From town center to shopping center: The reconfiguration of community marketplaces in postwar America. American Historical Review, 101(4), 1050-1081. doi: $10.2307 / 2169634$

Connell, J. (1999). Beyond Manila: Walls, malls, and private spaces. Environment and Planning A, 31(3), 417-439. doi: 10.1068/a310417

De Certeau, M. (1984) The practice of everyday life. Berkeley: University of California Press.

De Mattos, C. (1999). Santiago de Chile, globalización y expansión metropolitana: lo que existía sigue existiendo. EURE, 25(76), 29-56. doi: 10.4067/S0250-71611999007600002

Farías, I. (2009). Intimidad cultural en espacios de consumo. El Mall Plaza Vespucio y la imposibilidad de una cultura pública. En F. Pérez \& M. Tironi (Eds.), SCL. Espacios prácticos y cultura urbana (pp. 16-29). Santiago: ARQ Ediciones.

Fishman, R. (1987). Bourgeois utopias: The rise and fall of suburbs. Nueva York: Basic Books.

Gallardo, F. (1994). ¡Al fin! Todos somos posmodernos. La cultura material como paradigma de lo cotidiano. Mapocho, 35, 193-199. 
Galetovic, A., Poduje, I. \& Sanhueza, R. (2009). Malles en Santiago. De centros comerciales a centros urbanos. Estudios Públicos, 114 (otoño), 219-252. Disponible en http://www.cepchile.cl /dms/lang_1/doc_4432.html

García Canclini, N. (1999). Imaginarios urbanos. Buenos Aires: Eudeba.

Glaser, B. \& Strauss, A. (1967). The discovery of grounded theory. Chicago: Aldine.

Gorelik, A. (2004 [1994]). Miradas sobre Buenos Aires. Historia cultural y crítica urbana. Buenos Aires: Siglo XXI.

Goss, J. (1999). Once upon a time in the commodity world: An unofficial guide to mall of America. Annals of the Association of American Geographers, 89(1), 45-75. doi: 10.1111/0004 $-5608.00130$

Gruen, V. (1943). Shopping center. Architectural Forum, 78, 101-03.

Halpern, P. (2002). Los nuevos chilenos y las batallas por sus preferencias. Santiago: Planeta.

Hayden, D. (2002). Revisando la comedia suburbana. EURE, 28(83), 137-142. doi: 10.4067/S0250 $-71612002008300009$

Harris, M. (1979). El desarrollo de la teoría antropológica. Madrid: Siglo XXI.

Judd, D. (1995). The rise of the new walled cities. En H. Ligget \& D. Perry (Eds.), Spatial practices: Critical explorations in social/spatial theory (pp. 144-166). Thousands Oaks, CA: Sage.

Kim, Y., Kim, E., \& Kang, J. (2003). Teen's mall shopping motivation: functions of loneliness and media usage. Family and Consumer Sciences Research Journal, 32(2), 140-167. doi: 10.1177/1077727X03032002004

Lavín, J. (1987). Chile: La revolución silenciosa. Santiago: Zig-Zag.

Lefebvre. H. (1991). The production of space. Oxford: Blackwell Publishers.

Lefebvre, H. (1996). The right to the city. En Writings on cities (pp. 147-159). Cambridge: Blackwell. Versión electrónica PDF en http://www.columbia.edu/ jwp70/Teaching/v3565/LEFEB VRE_writings\%20on\%20cities.ch3+14_1968.pdf

Lewis, G. (1989). Rats and bunnies: Core kids in an American mall. Adolescence, 24(96), 881-889.

Liernur, F. (1991). Réquiem para la plaza y la fábrica. Nueva Sociedad, 114, 105-112. Disponible en http://www.nuso.org/upload/articulos/2016_1.pdf

Lieberman, T. (2004). Mallville: Mixing uses in the shopping center of the future. Tesis para optar al grado de Magíster en Planificación Urbana, Massachusetts Institute of Technology, Boston. No publicada.

Lofland, L. (1998). The public realm: Exploring the city's quintessential social territory. Nueva York: Aldine de Gruyter.

Magendzo, S. \& Bahamondes, M. (2005). “Los niños(as) en el espacio del centro comercial”. Investigación y Desarrollo, 13(1), 2-31. Disponible en http://redalyc.uaemex.mx /pdf/268/26813101.pdf

Matthews, H., Taylor, M., Percy-Smith, B., \& Limb, M. (2000). The unacceptable flaneur: The shopping mall as a teenage hangout. Childhood, 7(3), 279-294.

Marín, T. (2008). Nuevas formas de moralidad en los y las jóvenes chilenos. Última Década, 28, $143-$ 165. doi: 10.4067/S0718-22362008000100008

Merts, J. E. (1949). The shopping center: A new trend in retailing. Journal of Marketing, 13, 374-79.

Moulian, T. (1998). El consumo me consume. Santiago: LOM. 
Mugan, G. \& Erkip, F. (2009). Discrimination against teenagers in the mall environment: A case from Ankara, Turkey. Adolescence, 44(173), 209-232.

Nichols, J. C. (1948). Shopping centers: Look before you leap. Urban Land, 7, 1-.

Ortiz, S. (1994). Shopping for sociability in the mall. En D. A. Chekki (Ed.), Research in Community Sociology (Vol. 1, pp. 183-199). Greenwich, CT: JAI Press.

Perry, I. (2010). Demalling, remalling, it's all falling. Tesis para optar al grado de Magíster en Arquitectura. Massachusetts Institute of Technology, Boston. No publicada.

PNUD [Programa de Naciones Unidas para el Desarrollo]. (1998). Informe Desarrollo Humano en Chile. Las paradojas de la modernización. Santiago: Autor.

PNUD/INJUV [Programa de Naciones Unidas para el Desarrollo/Instituto Nacional de la Juventud, Chile]. (2003). Transformaciones culturales e identidad juvenil en Chile. Santiago: PNUD.

Rasse, A., Salcedo, R. \& Pardo, J. (2009). Transformaciones económicas y socioculturales: ¿Cómo segmentar a los chilenos hoy? En A. Joignant \& P. Güell (Coords.), El arte de clasificar a los chilenos. Enfoques sobre los modelos de estratificación en Chile (pp. 17-36). Serie Políticas Públicas. Santiago: Ediciones UDP (Universidad Diego Portales)/Universidad Alberto Hurtado. Recuperado de http://www.expansiva.cl/media/publicaciones/libros/pdf/10.pdf

Rile Hayward, C. (2001). Between the street and the mall: Social space and democratic possibility. Documento presentado en el Midwest Political Science Conference, Chicago, 19-22 abril.

Ritzer, G. (2003). Islands of the living dead: The social geography of McDonaldization. American Behavioral Scientist, 47, 119-136. doi: 10.1177/0002764203256179

Sack, R. (1988). The consumer's world: Place and context. Annals of the Association of American Geographers, 78(4), 642-664. doi:10.1111/j.1467-8306.1988.tb00236.x

Salcedo, R. (2002). El espacio público en el debate actual: Reflexiones críticas en torno al urbanismo post-moderno. EURE, 28(84), 5-19. doi: 10.4067/S0250-71612002008400001

Salcedo, R. (2003a). Lo local, lo global y el mall: La lógica de la exclusión y la interdependencia. Revista de Geografia Norte Grande, 30, 103-116.

Salcedo, R. (2003b). When the global meets the local at the mall. American Behavioral Scientist, 46(8), 1084-1103. doi: 10.1177/0002764202250500

Salcedo, R. \& Stillerman, J. (2010). Customers' spatial practices in a Santiago, Chile Mall: Control, agency and co-production. Manuscrito no publicado.

Sarlo, B. (1994). Escenas de la vida postmoderna. Buenos Aires: Ariel.

Sarlo, B. (2009). La ciudad vista. Mercancías y culturas urbanas. Buenos Aires: Siglo XXI.

Sato, A. (1981). Un simulacro urbano. Punto, 19(63), 25-35.

Staehely, L., \& Mitchel, D. (2006). USA's destiny? Regulating space and creating community in American shopping malls. Urban Studies, 43(5-6), 977-992. doi: 10.1080/00420980600676493

Stillerman, J., Salcedo, R., Phillips, G., \& Covarrubias, S. (2010). Middle class and teenage identity formation: Youth discourses and practices in a Santiago, Chile Mall. Manuscrito no publicado.

Stillerman, J. (2006). Private, parochial and public realms in Santiago, Chile's retail sector. City \& Community, 5(3): 293-316. doi: 10.1111/j.1540-6040.2006.00182.x

Thomas, I. (2006). The mall as a resort. Urban Land (August), 107-109. Disponible en http://www.docstoc .com/docs/71924473/Urban-Land-August-2006

Tironi, E. (1999). La irrupción de las masas y el malestar de las elites. Santiago: Grijalbo. 
Voyce, M. (2006). Shopping malls in Australia: The end of public space and the rise of 'consumerist citizenship'? Journal of Sociology, 42(3): 262-286. doi:10.1177/1440783306066727

Welch, K. C. (1948). Regional shopping centers: Some projects in the Northeast. Journal of the American Institute 